\title{
SURFACE TENSION OF SOAP SOLUTIONS AND ITS RELA- TION TO THE THICKNESS OF ADSORBED FILMS
}

\author{
By Ronald Bulkley and F. G. Bitner
}

\begin{abstract}
A sharp minimum in the surface tension-time curves of certain soap solutions has been interpreted in the literature as indicating the gradual formation of a surface film possessing the properties of a plastic solid and having a thickness equivalent to some 10,000 molecular lengths. In this paper the hypothesis is advanced that the formation of the thick film is brought about by chemical action between carbon dioxide from the air and dissolved soap. Surface tension experiments performed in the open air have confirmed the existence of the minimum point observed by previous investigators. However, when solutions prepared in exactly the same manner were tested by the same method in an atmosphere free from carbon dioxide no minimum point was found. The solutions behaved instead in the manner which is characteristic of colloidal solutions in general. These results support the hypothesis advanced above and leave the preponderance of evidence against the existence of a film of extraordinary thickness at the surface of soap solutions.
\end{abstract}

\section{CONTENTS}

I. Introduction

II. Experimental

III. Discussion

\section{INTRODUCTION}

In the course of an investigation ${ }^{1}$ to determine the limiting distance to which a solid surface can affect the viscosity of a contiguous liquid, one of the authors had occasion to review the experimental work reported in the literature on the viscosity of liquids at the airliquid interface. The most diverse conclusions as to the nature and magnitude of this property have been reached by different investigators even from the performance of the same experiment. Thus Plateau ${ }^{2}$ observed that the surface viscosity of some liquids was higher than the viscosity in bulk, while the surface viscosity of other liquids was lower than in bulk. His experiments were repeated by Luvini ${ }^{3}$ and, at a later date, by Lord Rayleigh, ${ }^{4}$ both of whom arrived at the conclusion that the anomalous surface viscosities observed by Plateau were due to contamination. Stables and Wilson ${ }^{5}$ measured the rate of damping of a torsion pendulum in the form of a thin disk suspended in the surface of a saponin solution, and found an abnormally high superficial viscosity.

1 The Viscosity of Liquids as Measured in Fine Capillaries, Dissertation of Ronald Bulkley for degree of Doctor of Philosophy at Johns Hopkins University; 1930.

2 Joseph A. F. Plateru, Statique des liquides, 8th series, 1873.

- Giovanni Luvini, On the Adbesion Between Solids and Liquids, Phil. Mag. (4), 40, pp. 190 to 197 ; 1870.

Lord Rayleigh, On the Superficial Viscosity of Water, Proc. Roy. Soc., 48, pp. 127 to 140; 1890

W. H. Stables and A. E. Wilson, On the Viscosity of a Solution of Saponine, Phil. Mag. (5), 15, pp. 406 to $414 ; 1883$. 
du Noüy, ${ }^{6}$ with much more delicate instruments, found for organic colloids, in general, that the surface viscosity increased and the surface tension decreased as a function of time when the measurements were made always on the same surface layer instead of on "a continuously renewed layer." Both phenomena were explained by assuming an increasing concentration of the suspended colloidal material in the surface due to adsorption. By taking observations on solutions of various concentrations a decided minimum value for the surface tension was discovered which occurred always at the same concentration for a given material. This minimum value was postulated as occurring when the surface of the containing vessels and the free liquid surface as well were just covered with an oriented monomolecular layer of the solute.

Perhaps the most remarkable results in the literature are those reported by Wilson and Ries. ${ }^{7}$ By several independent methods of attack these authors arrived at the conclusion that the surface of a soap solution is a film with the properties of a plastic solid, the thickness of the film increasing with time to reach a value of from 10 to $40 \mu$. This is of the order of 10,000 molecular lengths as contrasted with the monomolecular film postulated by du Noüy. Wilson and Ries worked with much higher concentrations than du Noüy. They found a sharp minimum and a sudden rise in the surface tensiontime curves for palmitate and stearate soaps, which they explained by assuming that the film has the properties of a plastic solid, and at a critical thickness the force necessary to overcome the increasing yield value becomes greater than the corresponding reduction of surface tension.

Wilson and Ries apparently were aware that carbon dioxide would react chemically with their soap solutions, but, as far as can be judged from their paper, their experiments were nevertheless conducted in open vessels exposed to the ordinary atmosphere of the laboratory. It is conceivable, therefore, that the film on their solutions was formed by complete or partial decomposition of the soap by carbon dioxide to give free acid or some of the numerous acid salts described by McBain. ${ }^{8}$ These probably would be lighter than the solution and would float at the surface, the thickness of the partially rigid layer increasing as the carbon dioxide diffused deeper into the liquid and the products of the reaction accumulated. Since the surface tension measurements are easy of performance it seemed worth while to test out this hypothesis by determining the shape of the surface tension-time curves when the solutions were at all times carfeully protected against contamination with carbon dioxide.

\section{EXPERIMENTAL}

The surface tension measurements were made with a du Noüy tensiometer. This instrument measures directly the force exerted by the surface film of the liquid under test upon a platinum ring of known mean circumference when the ring is pulled away from the liquid in which it has been dipped. The usual container used for the liquid

- P. L. du Nouly, A Note on the Surface Viscosity of Colloidal Solutions, Sci., 61, p. 117; 1925 . See also Surface Tension of Colloidal Solutions and Dimensions of Certain Organic Molecules, Colloidal Chem., 1, pp. 267 to 275 , Chemical Catalog Co.; 1926.

Robert E. Wilson and E. D. Ries, Surface Films as Plastic Solids, Coll. Sym. Mon., 1, pp. 145 to 173; 1923.

J. W. McBain, Colloidal Chemistry of Sosp, B. A, A, S. Third Report on Colloid Chemistry; 1920. 
with this instrument is an open vessel which exposes the liquid to the air, the composition of which varies from hour to hour and from day to day. Figure 1 shows some typical results of tests performed in open vessels under conditions similar to those of Wilson and Ries. The solutions were made by adding 1 part of sodium oleate, stearate or palmitate to 25,000 parts by weight of hot distilled water which had been boiled about 30 minutes to rid it of $\mathrm{CO}_{2}$. The solutions were then stoppered and allowed to cool. The surface tension-time curves are almost identical with those found by Wilson and Ries. The curve for the oleate shows the decline to steady value, those for the stearate and palmitate the rise after minimum. The stock solutions were protected from $\mathrm{CO}_{2}$ by passing all the air which entered the flask on cooling or while withdrawing samples through a soda-lime tube. All samples were withdrawn by a syphon from the center of the flask.

The container used in later experiments was a 3-necked Woulff bottle as shown in Figure 2. The tensiometer ring was suspended through the middle neck by an aluminum wire of sufficient diameter to give rigidity and of sufficient length to reach the bottom of the

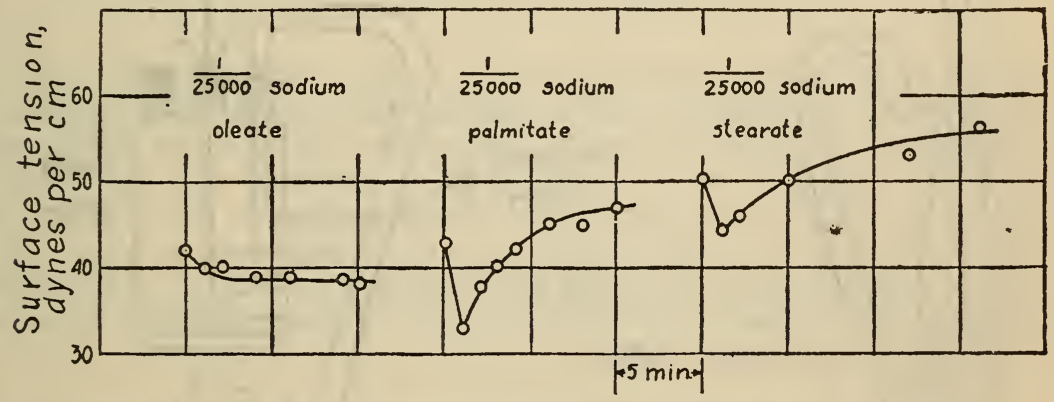

FIGURE 1.-Surface tension-time graphs for solutions in open vessels

bottle. This hole was then partly closed by a split-cork ring leaving a hole one-half centimeter in diameter through which the aluminum wire could move easily without touching the side. Air was supplied to the bottle through another neck by a glass tube which extended about halfway to the bottom. The solution was syphoned into the bottle through the third neck which was then stoppered. Air from the house pressure line was passed first through a wash bottle containing concentrated $\mathrm{H}_{2} \mathrm{SO}_{4}$ to indicate the rate of flow, through a drying tube containing $\mathrm{P}_{2} \mathrm{O}_{5}$ to remove moisture, and then to the Woulff bottle. When air which was free from $\mathrm{CO}_{2}$ was desired, a tube containing soda lime was introduced between the indicator and the drying tube. The air stream was allowed to run through the bottle for about 15 minutes before the sample was introduced. The purpose of this circulation was to rid the bottle of $\mathrm{CO}_{2}$-laden air.

The drying tube of $\mathrm{P}_{2} \mathrm{O}_{5}$ was used to insure approximately constant humidity in the Woulff bottle. That the initial decrease of surface tension with time was not due to evaporation from the surface was shown by making a few tests with the stearate and palmitate solutions while moist air was passed through the bottle. The air was moistened by bubbling through water near the boiling point and then through a column of distilled water about $50 \mathrm{~cm}$ deep which was kept 
at room temperature. Under these conditions both solutions showed the characteristic drop in surface tension with time.

With the apparatus in place, a stream of air regulated to about 200 bubbles per minute was passed into the Woulff bottle. Since this air passed from the bottle through the small hole around the aluminum wire, there was little chance of backward diffusion into the bottle.

The sample was syphoned into the bottle after allowing some of the solution to run to waste, thus obtaining a better sample of the solution at the center of the flask. The first measurement was made as quickly as possible, and succeeding ones at measured time intervals. The air stream was stopped for each measurement, an operation of about 20 seconds,

Enlorged detoil

Split Cork Ring
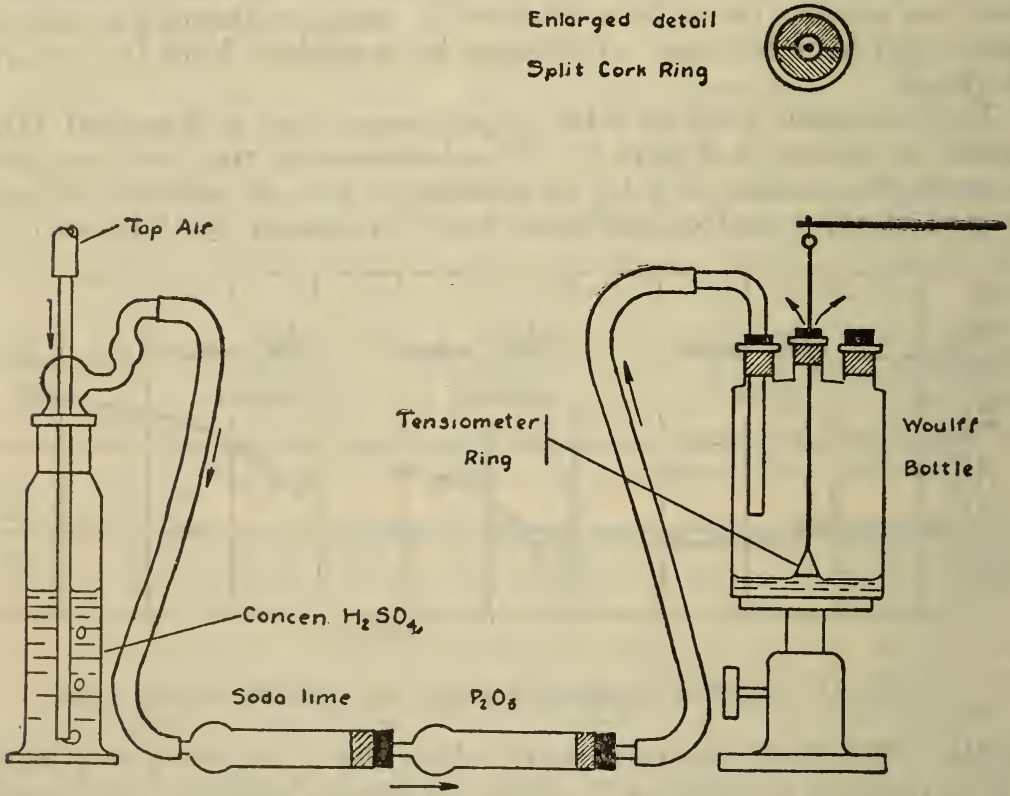

FIGURE 2.-Sketch of container and train for removing $\mathrm{CO}_{2}$ from air

Figures 3 and 4 show typical results of tests conducted in Woulff bottles on sodium-stearate solutions.

When the air stream had not been freed of $\mathrm{CO}_{2}$ the surface tensiontime curves were much the same as when made in an open vessel. (Figs. 3, $a$ and 4,a.) The chief difference noticed was a slower decrease in surface tension with time. When the air in contact with the soap solutions had been freed of $\mathrm{CO}_{2}$, the surface tension decreased at a slower rate as shown by Figures $3, b$ and $4, b$, reaching a steady value which held for about four hours or until the test was stopped. For the solution in Figure 4 , the soda-lime tube was removed after the surface tension had remained steady for about four hours. Measurements then made at 10-minute intervals showed a sharp minimum.

Solutions of sodium stearate of high purity, made in the laboratory by titrating an alcoholic solution of chemically pure stearic acid with an alcoholic solution of $\mathrm{NaOH}$, gave the same results as did the commercial product. 


\section{DISCUSSION}

The authors offer no explanation of the observations here reported other than the hypothesis advanced earlier in the paper. 'This hypothesis is set forth merely as a tentative explanation, and it is supported only qualitatively by the meager evidence we have described. If the reaction of carbon dioxide with sodium oleate in the surface layer of a solution should proceed far enough to produce oleic

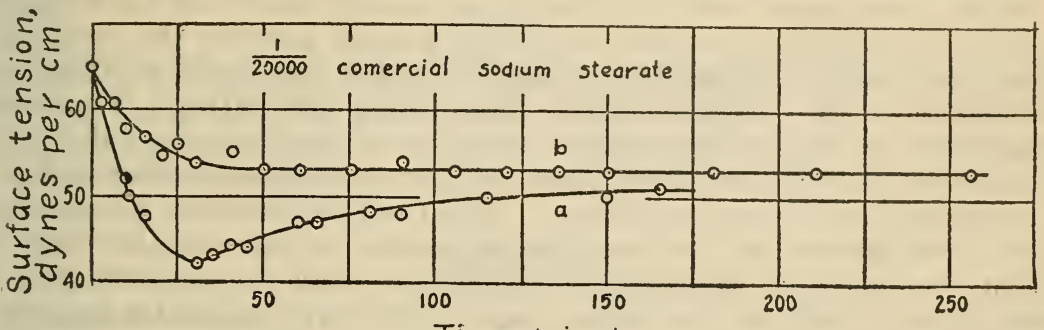

Time minutes

FIGURE 3.-Surface tension-time graphs for soap solution in Woulfj bottle $a, \mathrm{CO}_{2}$ not removed from air stream; $b, \mathrm{CO}_{2}$ removed by soda lime.

acid, the surface would be covered with a thin liquid film which would act only to lower the surface tension. No minimum in the surface tension-time curve would be expected for this solution exposed to the air and none was observed. On the other hand, the film resulting from the reaction of carbon dioxide with palmitate or stearate would be of a more or less solid nature and conceivably would acquire sufficient rigidity to produce the rise in the apparent surface tension which was found. In the absence of carbon dioxide all the solutions should

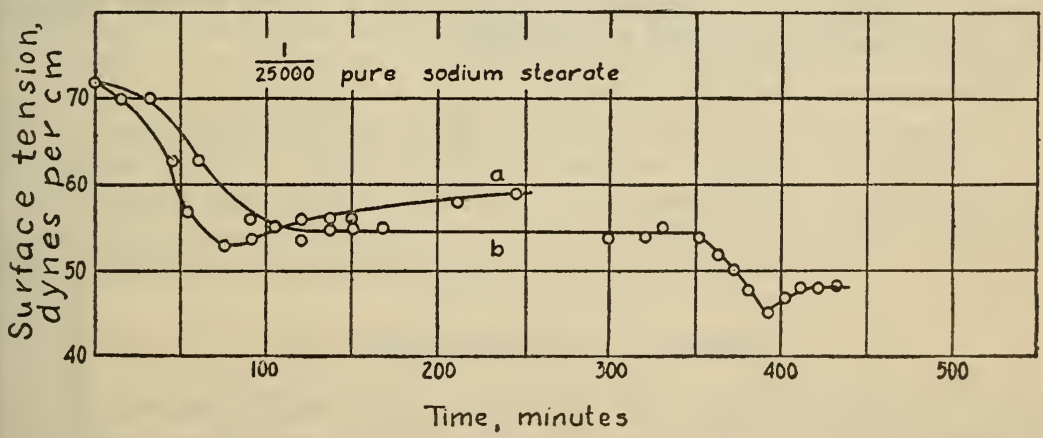

FIGURE 4.-Surface tension-time graphs for soap solution in Woulff bottle $a, \mathrm{CO}_{2}$ not removed from air stream; $b, \mathrm{CO}_{2}$ removed by soda lime.

give a gradually decreasing surface tension as the concentration of the soap in the surface increased by adsorption. When equilibrium has been established between adsorbed soap in the surface and dissolved soap in the body of the liquid the surface tension should reach a low steady value as was actually observed.

Many disturbing factors enter to complicate the results, such as the age of the solution, the degree of hydrolysis of the dissolved soap, etc. The absolute values probably do not have much significance. In the 
particular examples chosen here measurements made in the presence of carbon dioxide gave for commercial stearate a graph which is lower throughout its entire length than the corresponding graph for data taken in the absence of carbon dioxide. For pure sodiumstearate solutions the two curves crossed each other. Only the shape of the curves, that is, the relative values of the surface tension at different times for a single sample, are reliable as indicating the nature of the phenomena taking place. The one invariable observation which is common to all the graphs, including many not shown in this paper, is that the presence of carbon dioxide hastens the decrease in surface tension before the minimum point has been reached, and directly causes the rise which takes place thereafter. This seems sufficient to demonstrate that there is no sound basis for belief in the existence of a film of more than ordinary thickness on the surface of uncontaminated soap solutions. From ample evidence accumulated by investigators in the past the conclusion is unavoidable that this film, in which the properties are different from the properties of the solution in bulk, is not more than a very few molecular lengths in thickness.

Interesting results might be found by the further study of the surface tension of colloidal solutions by the capillary tube method or by other methods under carefully controlled conditions. Barring experimental difficulties the ideal situation would of course be to have the surface of the solution in contact only with its own vapor.

\section{CONCLUSIONS}

1. There is no minimum point in the surface tension-time curve of soap solutions protected from carbon dioxide.

2. Such solutions are probably covered not by a plastic film several thousand molecules in thickness but only by a much thinner adsorbed film, a few molecules thick at the most.

Washington, July 31, 1930. 